BULLETIN OF THE

AMERICAN MATHEMATICAL SOCIETY

Volume 78, Number 4, July 1972

\title{
NONSEPARATING FUNCTION ALGEBRAS
}

\author{
BY L. Q. EIFLER
}

Communicated by Felix Browder, December 29, 1971

Let $A$ be a function algebra on $X$ (compact). We say $A$ is a separating algebra on $X$ if for each closed subset $S$ of $X$ and for each $x \in X \backslash S$ there exists $f$ in $A$ such that $f(x)=0$ and $f$ does not vanish on $S$. We say that $A$ is essential on $X$ if for each open subset $U$ of $X$ there is a continuous function $f \notin A$ such that $f$ vanishes on $X / U$. Csordas and Reiter asked [2] if there exists a nonseparating, essential algebra $A$ on a (connected) space $X$ for which $X$ is the maximal ideal space of $A$ and also the Silov boundary of $A$. We give an example of such an algebra and simple examples of nonseparating algebras.

Given a compact subset $K$ of $C^{n}$, let $P(K)$ denote the uniform closure in $C(K)$ of the polynomials in $z_{1}, \ldots, z_{n}$. An easy application of Hurwitz' theorem [1, p. 176] shows that the first three of the following algebras are nonseparating.

ExAMPLE 1. Let $\Delta=\{z:|z| \leqq 1\}$. Then $P(\Delta \times \Delta)$ is nonseparating since $f(\Delta \times \Delta)=f(\{(z, w):|z|=1$ or $|w|=1\})$ for each $f$ in $P(\Delta \times \Delta)$. Also, $\Delta \times \Delta$ is the maximal ideal space of $P(\Delta \times \Delta)$.

EXAMPLE 2. The algebra $P([0,1] \times \Delta)$ is nonseparating since $f([0,1] \times \Delta)=f(\{(t, z): t=0$ or $|z|=1\})$. Also, $[0,1] \times \Delta$ is the maximal ideal space of $P([0,1] \times \Delta)$.

ExAmple 3. If $A$ is a separating algebra on $X=M(A)$ and if $B$ is a function algebra on $X$ containing $A$, then $B$ is separating on $X$ but not necessarily separating on $M(B)$. Let $B$ denote the uniform closure in $C(\Delta)$ of polynomials in $z$ and $|z|$. Then $P(\Delta) \subseteq B \subseteq C(\Delta)$. One can embed $B$ into $P([0,1] \times \Delta)$ by setting $F(r, z)=f(r z)$ for $0 \leqq r \leqq 1$ and $|z| \leqq 1$. Now one can see that the maximal ideal space of $B$ is $[0,1] \times \Delta /\{0\} \times \Delta$ and so $B$ is nonseparating on its maximal ideal space.

Example 4. Let $S^{2}=C \cup\{\infty\}$ and let $D=\{z:|z|<1\}$. Let

$$
B=\left\{f \in C\left(S^{2}\right): f \text { is analytic in } D\right\} \text {. }
$$

Then $M(B)=S^{2}$ and $f\left(S^{2}\right)=f\left(S^{2} \backslash D\right)$ for each $f$ in $B$. One easily checks that $S^{2}$ is the maximal ideal space of $B$. Fix $f$ in $B$ and assume $f$ does not vanish on $S^{2} \backslash D$. If $f$ has a zero in $D$, then there exist $\alpha_{1}, \ldots, \alpha_{n} \in D$ such that $g(z)=f(z) \prod_{k=1}^{n} z /\left(z-\alpha_{k}\right)$ belongs to $B$ and vanishes only at 0 . Given $\infty \geqq r>0$, define $\Gamma_{r}(\theta)=g\left(r e^{i \theta}\right)$. All of the curves $\Gamma_{r}$ are homotopic

AMS 1970 subject classifications. Primary $46 \mathrm{~J} 10$.

Key words and phrases. Function algebras. 
to each other in $C \backslash\{0\}$ and $\Gamma_{\infty}$ is constant. For $r$ small, the winding number of $\Gamma_{r}$ around 0 is equal to the order of the zero of $g$ at 0 . Hence, $f\left(S^{2}\right)=f\left(S^{2} \backslash D\right)$.

EXAMPLE 5 . We now construct an algebra $A$ on a space $X$ such that $A$ is nonseparating and essential and $X$ is the maximal ideal space and Silov boundary of $A$. (1) Choose $z_{i} \in C$ and $r_{i}>0$ such that $\sum r_{i}<\infty$, the discs $D_{i}=\left\{z:\left|z-z_{i}\right|<r_{i}\right\}$ are mutually disjoint and contained in $\Delta=\{z:|z| \leqq 1\}$, and $Y=\Delta \backslash \bigcup_{i=1}^{\infty} D_{i}$ has no interior. (2) Let $R(Y)$ denote the uniform closure in $C(Y)$ of the rational functions with poles off $Y$. Then $R(Y)$ is essential and $Y$ is the maximal ideal space and the Silov boundary of $R(Y)$. (3) Let $B$ be a nonseparating function on a compact metric space $K^{\prime}$ where $K^{\prime}=M(B)$ [Examples 1-4]. Let $K$ be a Cantor set of measure zero in $\{z:|z|=1\}$ and let $\rho$ be a continuous map of $K$ onto $K^{\prime}$. Now set $A=\{f \in R(Y): f \in B \circ \rho\}$. Since $K$ is a peak interpolation set for $R(Y)$, the space $Y / \rho$ is the maximal space of $A$. (See [3, Remark 2.2].) $A$ is essential and $Y / \rho$ is the Silov boundary of $A$. Finally, $A$ is nonseparating.

\section{REFERENCES}

1. L. V. Ahlfors, Complex analysis. An introduction to the theory of analytic functions of one complex variable, McGraw-Hill, New York, 1953. MR 14, 857.

2. G. Csordas and $\mathrm{H}$. Reiter, Separating function algebras (submitted).

3. I. Glicksberg, A remark on analyticity of function algebras, Pacific J. Math. 13 (1963), 1181-1185. MR 27 \#6151.

Department of Mathematics, Louisiana State University, Baton Rouge, Louisiana 70803. 